\title{
Tetrahydro-1,4-benzodiazepin-2-ones from Ugi MCR Intermediates
}

Gategory

Synthesis of

Heterocycles

\section{Key words}

tetrahydro-1,4benzodiazepin-2ones

Ugi reaction trimethylphosphine

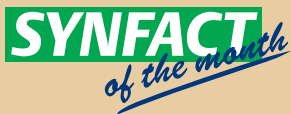

Significance: Tron and co-workers set to test the very interesting concept of setting a nucleophilic intramolecular trap for the intermediate Ugi imide, normally generated in a MCR reaction. The Ugi imides A were synthesized in a straightforward fashion and, after purification, subjected to a Staudinger reduction followed by heating, which resulted in the formation of 1,4-benzodiazepine-2one derivatives. Only trimethylphosphine was reported to effect the reduction as the corresponding iminophosphoranes generated from other phosphines were too stable to hydrolysis even at reflux. The yields achieved per step were usually good, around $70 \%$, giving rise to final product yields of $14-46 \%$. The substrate scope was reasonably well studied.
Comment: Benzodiazepines are widely appreciated for various biological activities, for example, antitumor and anti-HIV (S. Forso Mini-Rev. Med. Chem. 2010, 7, 68). Surprisingly, it appears that only three synthetic strategies for tetrahydro-1,4benzodiazepin-2-ones were reported prior to the current procedure. It is procedurally simple and utilizes mostly inexpensive starting materials. The only drawback of this procedure is the need for trimethylphosphine, which is somewhat expensive and has pyrophoric tendencies. Sadly, the scale of reactions performed was not evident either in the article or in the supporting information. 